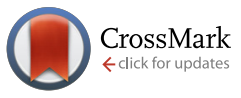

Cite this: Med. Chem. Commun., 2016, 7, 1973

\title{
Carbon monoxide attenuates the effects of snake venoms containing metalloproteinases with fibrinogenase or thrombin-like activity on plasmatic coagulation $\uparrow$
}

\author{
Vance G. Nielsen* and Charles M. Bazzell
}

\begin{abstract}
Exposure of plasma to iron and carbon monoxide (CO) renders fibrinogen resistant to fibrinogenolytic or thrombin-like activity contained in pit viper venom. However, the direct effects of iron/CO on venom activity are unknown. Thus, we assessed if four different, metalloproteinase containing snake venoms exposed to iron/CO or $\mathrm{CO}$ alone could attenuate their fibrinogenolytic or thrombin-like activity. Venom (0-500 $\mu \mathrm{g}$ $\mathrm{ml}^{-1}$ ) was exposed to 0-10 $\mu \mathrm{M} \mathrm{FeCl}_{3}$ and/or 0-100 $\mu \mathrm{M}$ carbon monoxide releasing molecule-2 (CORM-2), or inactivated CORM-2 (iCORM-2) for $3 \mathrm{~min}$ at room temperature. Venom solution (0-8 $\mu \mathrm{g} \mathrm{ml}^{-1}$ final concentration) was then placed in citrated human plasma containing tissue factor, followed by $\mathrm{CaCl}_{2}$ addition for commencement of coagulation. Data were determined with thrombelastography for 10-15 min at 37 ${ }^{\circ} \mathrm{C}$. Iron had no effect on the first venom tested, so only $\mathrm{CO}$ was investigated subsequently. Exposure of venom to $\mathrm{CO}$ attenuated fibrinogenolytic or thrombin-like activity, and iCORM-2 did not affect the venom activities. Further investigation of the effect of $\mathrm{CO}$ exposure on similar venoms is justified.
\end{abstract}

Received 15th June 2016,
Accepted 26th July 2016
DOI: 10.1039/c6md00336b
www.rsc.org/medchemcomm

\section{Introduction}

Envenomation by vipers with hemostatically active venom continues to be a major health problem worldwide. Systemic hypofibrinogenemia or afibrinogenemia can occur by the action of fibrinogenolytic enzymes that cleave fibrinogen such that it cannot be recognized and polymerized by thrombin, or by the action of thrombin-like or prothrombin activating enzymes that cause intravascular coagulation and consumption of available fibrinogen. Specifically, snake venom metalloproteinases (SVMP) may interact with the plasmatic coagulation several ways by acting as fibrinogenases, as thrombinlike enzymes, or as a prothrombin activators. ${ }^{1-5}$ Similarly, snake venom serine proteases (SVSP) also can affect coagulation as thrombin-like enzymes, or as activators of factor $\mathrm{V}^{6-8}$ For example, Crotalus and Agkistrodon pit viper species possess metalloproteinase-containing venoms that can be fibrinogenolytic, ${ }^{9-11}$ leading to consequent hypofibrinogenemia and hemorrhage after envenomation. Of interest, it has been demonstrated that pre-exposure of human plasma to small concentrations of iron and carbon monoxide (CO) causes plasma to be resistant to degradation of coagulation by Crotalus and Agkistrodon venoms containing either SVMP or SVSP. ${ }^{12-15}$ The putative mechanism for iron/CO mediated

Department of Anesthesiology, The University of Arizona College of Medicine, Tucson, Arizona, USA. E-mail: vnielsen@anesth.arizona.edu

$\dagger$ The authors declare no competing interests. protection was that these agents change the configuration of fibrinogen, making molecular recognition of catalytic sites by venom less favorable, ${ }^{12-15}$ based on the changes in ultrastructure of plasma clots mediated by iron/CO. ${ }^{16}$ In sum, the addition of iron/CO to plasma appears to indirectly attenuate the effects of fibrinogenase activity from a variety of species of venomous snakes on coagulation.

Of interest, the direct effects of iron/CO on snake venom in isolation were not determined in these previous works. ${ }^{12-15}$ Thus, when preliminary experiments to verify as a negative control that iron/CO would not inhibit the hemostatic effects of SVMP containing venom demonstrated the opposite effect, the decision to formally test this serendipitous finding was made. Thus, the purpose of this investigation was to determine if exposure of isolated SVMP containing venoms to iron and/or CO diminish fibrinogenolysis or abnormal procoagulation in human plasma.

\section{Materials and methods}

\section{Materials}

Lyophilized venom containing SVMP obtained from four different species of snakes were analyzed. The venoms and SVMP are displayed in Table 1. Crotalus atrox (western diamondback rattlesnake) and Agkistrodon contortrix mokasen (northern copperhead) venoms were obtained from the National Natural Toxins Research Center at Texas A\&M 
Table 1 Characteristics of metalloprotease containing snake venoms assessed

\begin{tabular}{lll}
\hline Species & Metalloproteinase/serine protease & \\
\hline Crotalus atrox & Metalloproteinase with fibrinogenase activity & Ref. \\
Agkistrodon contortrix mokasen & Metalloproteinase with fibrinogenase activity & 2 \\
Daboia russelli & Factor X activating metalloproteinase & 3 \\
& Prothrombin activating metalloproteinases & \\
& Serine protease with thrombin-like activity & 4,6 and 8 \\
Bothrops asper & Factor V activating serine protease & \\
& Prothrombin activating metalloproteinase & \\
& Serine protease with thrombin-like activity &
\end{tabular}

University-Kingsville, TX, USA. Daboia russelli (Russell's viper) venom was obtained from Sigma-Aldrich, Saint Louis, MO, USA. Bothrops asper (Fer-de-lance) venom was a generous gift from Sergio Bárcenas-Arriaga, graduate student of Alejandro Alagón of the Instituto de Biotecnología, Universidad Nacional Autónoma de México, Cuernavaca, Morelos, México. Venoms were reconstituted in calcium-free phosphate buffered saline (PBS, Sigma-Aldrich) at concentrations of 20-50 $\mathrm{mg} \mathrm{ml} \mathrm{m}^{-1}$, and stored at $-80{ }^{\circ} \mathrm{C}$. Pooled human normal plasma anticoagulated with sodium citrate was obtained from George King Bio-Medical, Overland Park, KS, USA. Ferric chloride $\left(\mathrm{FeCl}_{3}, 99.9 \%\right.$ pure), tricarbonyldichlororuthenium(II) dimer (CORM-2), and dimethyl sulfoxide (DMSO) were obtained from Sigma-Aldrich. The final concentrations of $\mathrm{FeCl}_{3}$ (dissolved in PBS) and CORM-2 (dissolved in DMSO) were 0$10 \mu \mathrm{M}$ and $0-100 \mu \mathrm{M}$, respectively; these were the concentrations used in previous works involving the effects of modifying fibrinogen prior to venom exposure in plasma. ${ }^{12-15}$ To isolate the effects of CO from the carrier molecule of CORM2, a $100 \mu \mathrm{M}$ solution of CORM-2 was placed in a sealed plastic tube and incubated in a to a $37^{\circ} \mathrm{C}$ water bath for 18 hours in order to release $\mathrm{CO}$ and become inactive (iCORM-2) as has been previously described. ${ }^{17}$

\section{Isolated fibrinogenase containing venom exposure experiments}

To increase the detection of effects of iron and/or CO on venom activity, the concentration of $C$. atrox and $A$. c. mokasen venoms chosen for isolated exposure and for addition to plasma was twice the amount expected to eliminate or enhance coagulation based on previous works. ${ }^{12,13}$ In the case of $C$. atrox venom, to test the effects of iron and CO (together and separately) exposure on SVMP fibrinogenase activity, a $1 \mathrm{ml}$ quantity of a $200 \mu \mathrm{g} \mathrm{m} \mathrm{m}^{-1}$ solution of venom in PBS was prepared for each condition subsequently presented. The conditions were: 1) $1 \%$ addition (v/v) of PBS and DMSO to PBS without venom; 2) $1 \%$ addition (v/v) of PBS and DMSO to PBS with venom; 3) $1 \%$ addition of $\mathrm{FeCl}_{3}$ and CORM-2 to PBS without venom; 4) $1 \%$ addition of $\mathrm{FeCl}_{3}$ and CORM-2 to PBS with venom; 5) $1 \%$ addition of $\mathrm{FeCl}_{3}$ and DMSO to PBS with venom; 6) $1 \%$ addition of CORM-2 to PBS with venom; and lastly, 7) 1\% addition of PBS and iCORM-2 to PBS with venom. As this first series of experiments demonstrated a significant difference in C. atrox venom effects on coagulation after $\mathrm{CO}$ exposure, only $\mathrm{CO}$ exposure experiments were conducted with the remaining venoms. Thus, in the case of A. c. mokasen venom, the effects of CORM-2 and iCORM-2 addition to $1 \mathrm{ml}$ of a $500 \mu \mathrm{g} \mathrm{ml}^{-1}$ solution of venom in PBS were tested and compared to the aforementioned conditions 1 and 2 . After $3 \mathrm{~min}$ of incubation at room temperature, $3.6 \mu \mathrm{l}$ of solution from one of these conditions was placed into $326 \mu \mathrm{l}$ of plasma and $10 \mu \mathrm{l}$ of tissue factor reagent $(0.1 \%$ final concentration in distilled water; Diagnostica Stago S.A.S., Asnieres sur Seine, France) in a disposable cup in a computer-controlled thrombelastograph $\AA$ hemostasis system (Model 5000, Haemonetics Inc., Braintree, MA, USA). After test solution addition, $20 \mu \mathrm{l}$ of $200 \mathrm{mM} \mathrm{CaCl}_{2}$ was immediately added to initiate clotting. Data were collected at $37{ }^{\circ} \mathrm{C}$ for $15 \mathrm{~min}$. The following parameters previously described ${ }^{12-15}$ were determined: time to maximum rate of thrombus generation (TMRTG): this is the time interval (min) observed prior to maximum speed of clot growth; maximum rate of thrombus generation (MRTG): this is the maximum velocity of clot growth observed (dynes per $\mathrm{cm}^{-2} \mathrm{~s}^{-1}$ ); and total thrombus generation (TTG, dynes per $\mathrm{cm}^{-2}$ ), the final viscoelastic resistance observed after clot formation.

\section{Isolated thrombin-like activity containing venom exposure experiments}

In the case of the two thrombin-like activity containing venoms, a final concentration of $2 \mu \mathrm{g} \mathrm{ml}^{-1}$ was used to produce a vigorous procoagulant response based on unpublished, preliminary work. The effects on D. russelli and B. asper venoms of $1 \%$ additions of solutions of PBS/DMSO, CORM-2 and iCORM-2 to $1 \mathrm{ml}$ of a $500 \mu \mathrm{g} \mathrm{ml}{ }^{-1}$ solution of venom in PBS were tested and compared to each other and to PBS/DMSO solution addition to PBS without venom present as outlined previously. After $3 \mathrm{~min}$ of incubation at room temperature, $3.6 \mu \mathrm{l}$ of solution from one of these conditions was placed into $336 \mu \mathrm{l}$ of plasma in a disposable cup in a computer-controlled thrombelastograph ${ }^{\circledR}$ hemostasis system (Model 5000, Haemonetics Inc.). After test solution addition, $20 \mu \mathrm{l}$ of $200 \mathrm{mM} \mathrm{CaCl}$ was immediately added to initiate clotting. Thrombelastographic parameter data were collected at $37^{\circ} \mathrm{C}$ for $10 \mathrm{~min}$ as previously described. The rationale for using only a $10 \mathrm{~min}$ time period to collect data was that least 13 to $15 \mathrm{~min}$ are required for contact activation of cup and pin with recalcified plasma is required to generate 
thrombin and generate measurable coagulation kinetic phenomena in our system.

\section{Determination of CORM-2 concentration SVMP activity relationship}

In order to determine the kinetic nature of SVMP response to various concentrations of $\mathrm{CO}$, we chose to focus on $\mathrm{CO}$ mediated modulation of $C$. atrox fibrinogenase activity. The six conditions ( $n=4$ per condition) tested were addition of $1 \%$ $(\mathrm{v} / \mathrm{v})$ of $0,10,20,30,40$, or $50 \mu \mathrm{M}$ CORM-2 to PBS with venom that was incubated for $3 \mathrm{~min}$ at room temperature as in the previous experiment series. Thereafter, $3.6 \mu \mathrm{l}$ of solution from one of these six conditions was placed into $326 \mu \mathrm{l}$ of plasma and $10 \mu \mathrm{l}$ of tissue factor reagent $(0.1 \%$ final concentration; Diagnostica Stago S.A.S.) in a disposable cup in a computer-controlled thrombelastograph ${ }^{\circledR}$ hemostasis system (Model 5000, Haemonetics Inc.). Subsequently, $20 \mu \mathrm{l}$ of 200 $\mathrm{mM} \mathrm{CaCl}_{2}$ was added to initiate clotting. Thrombelastographic data were collected at $37^{\circ} \mathrm{C}$ for $15 \mathrm{~min}$.

\section{Statistical analyses}

Data are presented as median (1st-3rd quartiles) or as raw data with kinetic modeling. All conditions were represented by $n=6$ replicates. This number of replicates were used as a statistical power $>0.8$ has been observed with similar analyses with this in vitro model. ${ }^{9,10}$ If no coagulation occurred, the sample observed was assigned a TMRTG value of 10 or 15 min depending on the experimental series, with values for MRTG and TTG designated as 0 . Thus, as the data were not normally distributed, Kruskall-Wallis one-way analysis of variance followed by Student-Newman-Keuls post hoc test were used for analyses (SigmaStat 3.1, Systat Software, Inc., San Jose, CA, USA). With regard to coagulation kinetic modeling of the C. atrox CORM-2 concentration fibrinogenase activity response relationship, data were analyzed with and graphed with a commercial graphics program (OrigenPro 7.5, OrigenLab Corporation, Northampton, MA, USA). $P<0.05$ was considered significant.

\section{Results and discussion}

Coagulation kinetic data for the four venoms tested are displayed in Tables 2-5. As seen in Table 2, compared to plasma with addition of PBS/DMSO, the further addition of C. atrox venom resulted in a significant, and essentially complete lack of coagulation. Addition of PBS containing $\mathrm{FeCl}_{3} /$ CORM-2 resulted in coagulation kinetic parameters not significantly different from plasma exposed to PBS/DMSO, but significantly different from samples exposed to C. atrox venom. Similarly, addition of PBS with $C$. atrox venom exposed to $\mathrm{FeCl}_{3} / \mathrm{CORM}-2$ resulted in coagulation kinetic parameters not significantly different from plasma exposed to PBS/DMSO, but significantly different from samples exposed to $C$. atrox venom. When PBS with $C$. atrox venom exposed to $\mathrm{FeCl}_{3}$ was tested, absence of coagulation not different from samples exposed to venom alone was observed. In contrast, PBS containing $C$. atrox venom exposed to CORM-2 added to plasma produced coagulation kinetic values not different from PBS/DMSO exposed plasma, but significantly different from plasma mixed with venom with PBS/DMSO exposure. Lastly, addition of PBS with C. atrox venom exposed to iCORM-2 to plasma resulted in no discernable coagulation, significantly different from the results observed with PBS/ DMSO exposed plasma but not different from plasma samples containing $C$. atrox venom with PBS/DMSO exposure.

With regard to $A$. c. mokasen venom coagulation kinetic data, as seen in Table 3, venom significantly prolonged TMRTG, decreased MRTG and decreased TTG compared to PBS/DMSO exposed plasma. However, addition of $A$. $c$. mokasen venom exposed to CORM-2 to plasma resulted in coagulation kinetics not significantly different from plasma

Table 2 Effects of $\mathrm{FeCl}_{3}, \mathrm{CORM}-2$ and iCORM-2 on C. atrox venom fibrinogenolysis in plasma

Effects of combinations of vehicle, venom and $\mathrm{FeCl}_{3}+\mathrm{CORM}-2$ on coagulation

\begin{tabular}{llll}
\hline Condition & TMRTG & MRTG & TTG \\
\hline DMSO/PBS vehicle & $3.7(3.2,3.8)$ & $4.7(4.1,5.3)$ & $168(153,174)$ \\
Venom & $15.0(15.0,15.0)^{a}$ & $0.0(0.0,0.0)^{a}$ & $0(0,1)^{a}$ \\
$\mathrm{FeCl}_{3}+\mathrm{CORM-2}$ & $3.7(3.2,3.8)^{b}$ & $5.0(4.8,5.2)^{b}$ & $168(165,176)^{b}$ \\
Venom $^{b} \mathrm{FeCl}_{3}+\mathrm{CORM-2}$ & $3.2(3.0,3.3)^{b}$ & $5.2(4.9,5.6)^{b}$ & $176(169,182)^{b}$
\end{tabular}

Effects of $\mathrm{FeCl}_{3}$ exposed venom on coagulation

\begin{tabular}{llll}
\hline Venom $+\mathrm{FeCl}_{3}$ & $15.0(15.0,15.0)^{a}$ & $0.0(0.0,0.0)^{a}$ & $0(0,0)^{a}$
\end{tabular}

Effects of CORM-2 or inactivated CORM-2 (iCORM-2) exposed venom on coagulation

\begin{tabular}{llll}
\hline Venom + CORM-2 & $3.0(3.0,3.1)^{a, b}$ & $5.9(5.7,6.1)^{a, b}$ & $172(168,178)^{b}$ \\
Venom + iCORM-2 & $15.0(15.0,15.0)^{a, c}$ & $0.0(0.0,0.0)^{a, c}$ & $0.0(0.0,0.0)^{a, c}$
\end{tabular}

Coagulation was activated with tissue factor addition to the samples in all conditions. DMSO/PBS vehicle $=1 \%$ addition of each of these vehicles to PBS solution without venom addition; venom $=2 \mu \mathrm{g} \mathrm{ml} \mathrm{m}^{-1}$ final concentration of $C$. atrox venom in DMSO/PBS vehicle solution; $\mathrm{FeCl}_{3}+\mathrm{CORM}-2=\mathrm{PBS}$ solution with $10 \mu \mathrm{M} \mathrm{FeCl}_{3}$ and $100 \mu \mathrm{M}$ CORM-2 final concentration; venom $+\mathrm{FeCl}_{3}+\mathrm{CORM}^{2}=\mathrm{venom} 2 \mu \mathrm{g} \mathrm{ml}^{-1}$ final concentration exposed to $10 \mu \mathrm{M} \mathrm{FeCl}_{3}$ and $100 \mu \mathrm{M}$ CORM-2; venom $+\mathrm{FeCl}_{3}=$ venom $2 \mu \mathrm{g} \mathrm{ml}{ }^{-1}$ final concentration exposed to $10 \mu \mathrm{M}$ FeCl 3 ; venom + CORM-2 = venom $2 \mu \mathrm{g} \mathrm{ml}^{-1}$ final concentration exposed to $100 \mu \mathrm{M}$ CORM-2; venom + iCORM- $2=$ venom $2 \mu \mathrm{g} \mathrm{ml}^{-1}$ final concentration exposed to $100 \mu \mathrm{M}$ iCORM-2. ${ }^{a} P<0.05$ vs. DMSO/PBS vehicle. ${ }^{b} P<0.05$ vs. venom. ${ }^{c} P<0.05 v s$. venom + CORM-2. 
Table 3 Effects of CORM-2 and iCORM-2 on A. c. mokasen venom fibrinogenolysis in plasma

\begin{tabular}{llll}
\hline Condition & TMRTG & MRTG & TTG \\
\hline DMSO/PBS vehicle & $3.7(3.2,3.8)$ & $4.7(4.1,5.3)$ & $168(153,174)$ \\
Venom & $7.6(7.1,8.1)^{a}$ & $1.2(1.0,1.4)^{a}$ & $100(88,114)^{a}$ \\
Venom + CORM-2 & $3.6(3.4,3.6)^{b}$ & $4.6(4.2,4.8)^{b}$ & $170(175,186)^{b}$ \\
Venom + iCORM-2 & $15.0(12.4,15.0)^{a, b, c}$ & $0.0(0.0,0.4)^{a, b, c}$ & $0(0,26)^{a, b, c}$
\end{tabular}

Coagulation was activated with tissue factor addition to the samples in all conditions. DMSO/PBS vehicle $=1 \%$ addition of each of these vehicles to PBS solution without venom addition; venom $=8 \mu \mathrm{g} \mathrm{ml}^{-1}$ final concentration of $A$. $c$. mokasen venom in DMSO/PBS vehicle solution; venom + CORM-2 = venom $8 \mu \mathrm{g} \mathrm{ml}{ }^{-1}$ final concentration of venom exposed to $100 \mu \mathrm{M} \mathrm{CORM-2}$; venom + iCORM-2 = venom $8 \mu \mathrm{g}$ ml ${ }^{-1}$ final concentration of venom exposed to $100 \mu \mathrm{M}$ iCORM-2. ${ }^{a} P<0.05$ vs. DMSO/PBS vehicle. ${ }^{b} P<0.05$ vs. venom. ${ }^{c} P<0.05$ vs. venom + CORM-2.

Table 4 Effects of CORM-2 and iCORM-2 on D. russelli venom thrombin-like activity in plasma

\begin{tabular}{llll}
\hline Condition & TMRTG & MRTG & TTG \\
\hline DMSO/PBS vehicle & $10.0(10.0,10.0)$ & $0.0(0.0,0.1)$ & $1(0,2)$ \\
Venom & $1.2(1.2,1.2)^{a}$ & $10.5(10.0,11.0)^{a}$ & $179(172,184)^{a}$ \\
Venom + CORM-2 & $3.3(3.2,3.6)^{a, b}$ & $4.8(4.4,5.5)^{a, b}$ & $144(134,152)^{a, b}$ \\
Venom + iCORM-2 & $1.3(1.2,1.3)^{a, c}$ & $10.0(9.5,10.5)^{a, c}$ & $184(176,187)^{a, c}$
\end{tabular}

$\mathrm{DMSO} / \mathrm{PBS}$ vehicle $=1 \%$ addition of each of these vehicles to PBS solution without venom addition; venom $=2 \mu \mathrm{g} \mathrm{ml} \mathrm{l}^{-1}$ final concentration of D. russelli venom in DMSO/PBS vehicle solution; venom + CORM-2 = venom $2 \mu \mathrm{g} \mathrm{ml}{ }^{-1}$ final concentration of venom exposed to $100 \mu \mathrm{M}$ CORM2 ; venom + iCORM-2 $=$ venom $2 \mu \mathrm{g} \mathrm{ml}^{-1}$ final concentration of venom exposed to $100 \mu \mathrm{M}$ iCORM-2. ${ }^{a} P<0.05 v s$. DMSO/PBS vehicle. ${ }^{b} P<$ 0.05 vs. venom. ${ }^{c} P<0.05$ vs. venom + CORM-2.

Table 5 Effects of CORM-2 and iCORM-2 on B. asper venom thrombin-like activity in plasma

\begin{tabular}{llll}
\hline Condition & TMRTG & MRTG & \multicolumn{1}{c}{ TTG } \\
\hline DMSO/PBS vehicle & $10.0(10.0,10.0)$ & $0.0(0.0,0.1)$ & $1(0,2)$ \\
Venom & $1.2(1.2,1.3)^{a}$ & $10.3(9.7,11.0)^{a}$ & $178(168,184)^{a}$ \\
Venom + CORM-2 & $4.4(4.2,4.8)^{a, b}$ & $4.2(4.0,4.4)^{a, b}$ & $128(124,131)^{a, b}$ \\
Venom + iCORM-2 & $2.1(2.1,2.2)^{a, b, c}$ & $9.0(8.7,9.2)^{a, b, c}$ & $175(172,182)^{a, c}$
\end{tabular}

$\mathrm{DMSO} / \mathrm{PBS}$ vehicle $=1 \%$ addition of each of these vehicles to PBS solution without venom addition; venom $=2 \mu \mathrm{g} \mathrm{ml} \mathrm{l}^{-1}$ final concentration of B. asper venom in DMSO/PBS vehicle solution; venom + CORM-2 = venom $2 \mu \mathrm{g} \mathrm{ml}{ }^{-1}$ final concentration of venom exposed to $100 \mu \mathrm{M}$ CORM- 2 ; venom + iCORM-2 = venom $2 \mu \mathrm{g} \mathrm{ml}^{-1}$ final concentration of venom exposed to $100 \mu \mathrm{M}$ iCORM-2. ${ }^{a} P<0.05 v s$. DMSO/PBS vehicle. ${ }^{b} P<0.05$ vs. venom. ${ }^{c} P<0.05$ vs. venom + CORM-2.

exposed to PBS/DMSO. Lastly, and surprisingly, addition of $A$. c. mokasen venom exposed to iCORM-2 to plasma resulted in essentially absent coagulation, with TMRTG, MRTG and TTG values significantly different from the other three conditions.

As for D. russelli venom with thrombin-like activity exposed to PBS/DMSO, as seen in Table 4, venom significantly decreased TMRTG, increased MRTG and increased TTG compared to PBS/DMSO exposed plasma, which was not capable of forming clot within $10 \mathrm{~min}$. After exposing D. russelli venom to CORM-2, the venom induced a significantly longer TMRTG, smaller MRTG and smaller TTG values in plasma compared to venom not exposed to CORM-2. Lastly, after being exposed to iCORM-2, D. russelli venom displayed coagulation kinetics in plasma not significantly different from venom exposed to PBS/DMSO but significantly different from plasma with addition of venom exposed to CORM-2 or after addition of PBS/DMSO without venom.

The data concerning the effects of exposure of $B$. asper venom to CORM-2 and iCORM-2 are displayed in Table 5 . The thrombin-like activity of $B$. asper venom exposed to PBS/
DMSO demonstrates significantly smaller TMRG, greater MRTG and greater TTG values that that observed with plasma without venom addition. Exposure of $B$. asper venom to CORM-2 resulted in significantly greater TMRTG, smaller MRTG and smaller TTG values in plasma compared to venom exposed to PBS/DMSO. Finally, exposure of B. asper venom to iCORM-2 resulted in TMRTG values significantly greater than venom exposed to PBS/DMSO but significantly smaller than values in plasma with venom exposed to CORM-2. However, MRTG and TTG values in plasma were not significantly different between iCORM-2 and PBS/DMSO exposed B. asper venom additions.

Lastly, with regard to the coagulation kinetic modeling of the $C$. atrox CORM-2 concentration fibrinogenase activity response relationship, the results of these experiments are displayed in Fig. 1-3. Exposure of $C$. atrox venom to progressively greater concentrations of CORM-2 resulted in significant, concentration-dependent decreases in TMRTG values, increases in MRTG values and increases in TTG values that were best described as sigmoidal relationships. 


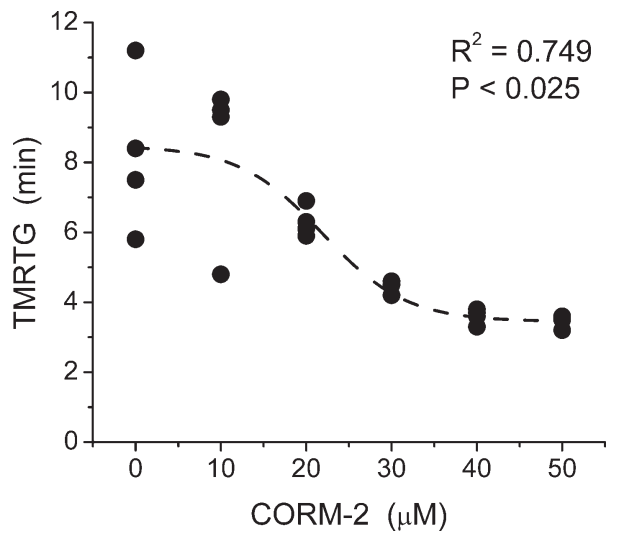

Fig. 1 Relationship between TMRTG and CORM-2 after exposure of $C$. atrox venom to various concentrations of CORM-2. Raw data for six concentrations ( $n=4 /$ concentration) of CORM-2 exposure of $C$. atrox venom are depicted. Dashed line represents kinetic modeled relationship, with coefficient of determination $\left(R^{2}\right)$ and statistical significance of the relationship displayed. The equation describing this relationship is as follows:

$$
\operatorname{TMRTG}(\min )=\frac{8.47}{1+\mathrm{e}^{(([\operatorname{CORM}-2(\mu \mathrm{M})]-21.9) 4.89)}} .
$$

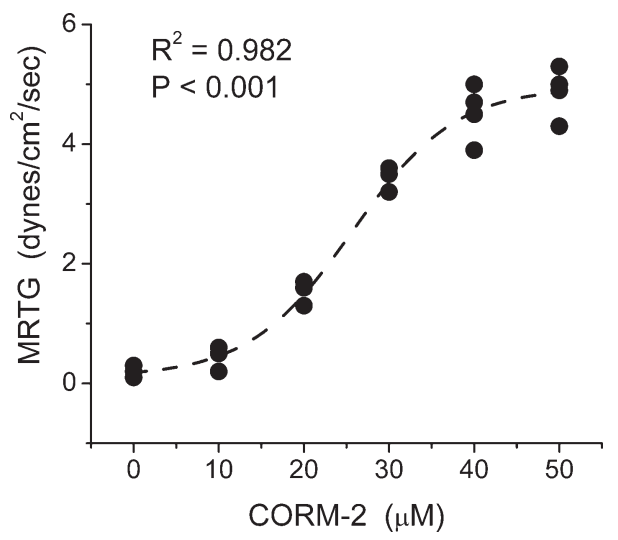

Fig. 2 Relationship between MRTG and CORM-2 after exposure of $C$. atrox venom to various concentrations of CORM-2. Raw data for six concentrations ( $n=4$ /concentration) of CORM-2 exposure of $C$. atrox venom are depicted. Dashed line represents kinetic modeled relationship, with coefficient of determination $\left(R^{2}\right)$ and statistical significance of the relationship displayed. The equation describing this relationship is as follows:

$$
\operatorname{MRTG}\left(\text { dynes per } \mathrm{cm}^{2} \text { per s }\right)=\frac{9.80}{1+\mathrm{e}^{(([\text {CORM-2 }(\mu \mathrm{M})]-25.6) 6.10)}} \text {. }
$$

The primary finding of the present investigation was that CO derived from CORM-2 significantly inhibited the fibrinogenolytic and procoagulant effects of two

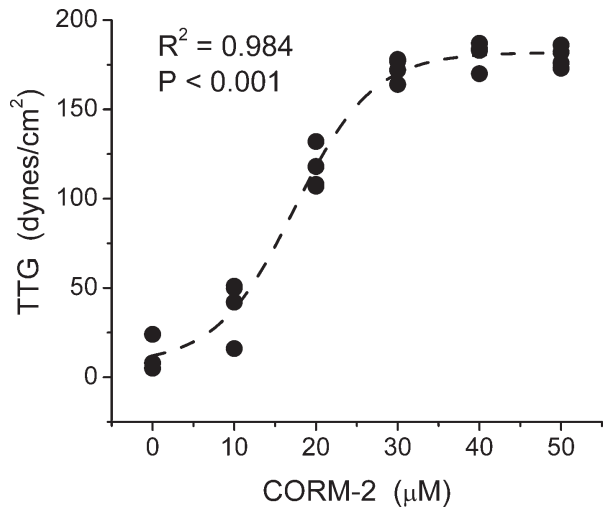

Fig. 3 Relationship between TTG and CORM-2 after exposure of $C$. atrox venom to various concentrations of CORM-2. Raw data for six concentrations ( $n=4 /$ concentration) of CORM-2 exposure of $C$. atrox venom are depicted. Dashed line represents kinetic modeled relationship, with coefficient of determination $\left(R^{2}\right)$ and statistical significance of the relationship displayed. The equation describing this relationship is as follows:

$$
\text { TTG }\left(\text { dynes per } \mathrm{cm}^{2}\right)=\frac{356}{1+\mathrm{e}^{(([\text {CORM-2 }(\mu \mathrm{M})]-17.4) 4.80)}} \text {. }
$$

fibrinogenolytic metalloproteinase and two thrombin-like metalloproteinase containing venoms, respectively. As iron had no effect on C. atrox venom, we tested the effects of CO and the carrier molecule, iCORM-2. CO derived from CORM2 has only once been demonstrated to directly inhibit the activity of metalloproteinases 1 and 2 in a human alveolar epithelial cell line. ${ }^{18}$ These authors speculated that CO, being known to bind to transition metals, ${ }^{19,20}$ could have bound to the $\mathrm{Zn}^{2+}$ metal located in the catalytic center of the enzyme as the mechanism of inhibition. ${ }^{18}$ However, $\mathrm{Zn}^{2+}$ binds to $\mathrm{CO}$ with less avidity than other transition metals. ${ }^{21}$ Of interest, CO has been shown to modulate protein function in an amino acid-dependent, heme-independent fashion; ${ }^{22}$ thus, perhaps CO may inhibit SVMP by binding to amino acid residues within or near the catalytic site of the enzyme, displacing the $\mathrm{Zn}^{2+}$ ion entirely. While the precise molecular mechanism by which CO inhibits SVMP remains to be elucidated, it can be inferred that such enzyme inhibition is CO concentration-dependent and sigmoidal in nature in the case of $C$. atrox venom fibrinogenolytic activity, not unlike that seen with heme-containing proteins. Given that the $\mathrm{Zn}^{2+}$ ion contained in SVMP have been demonstrated to be critical to ligand binding via molecular dynamic simulation, ${ }^{23}$ and that most of these enzymes possess $\mathrm{Zn}^{2+}$ containing catalytic centers ${ }^{1}$ the findings of the present study may have broad implications. For example, in addition to hemostatic effects, snake venom $\mathrm{Zn}^{2+}$ metalloproteinases also induce hemorrhage and circulatory failure. ${ }^{1}$ If $\mathrm{CO}$ inactivates a significant number of such snake venom $\mathrm{Zn}^{2+}$ metalloproteinases, then a new potential therapeutic direction for the treatment of envenomation may be possible. 
In addition to the changes in SVMP activity made by carbon monoxide exposure, two of the venoms demonstrated modifications by exposure to iCORM-2. Specifically, iCORM-2 enhanced $A$. $c$. mokasen venom fibrinogenase activity (Table 3 ) and inhibited $B$. asper venom thrombin-like activity (Table 5). The ruthenium dimer may be interacting with $\mathrm{Zn}^{2+}$ in the catalytic site of the SVMP or binding elsewhere on the enzyme in the absence of CO presumably interacting with the $\mathrm{Zn}^{2+}$. Our data indicate that in the presence of both released $\mathrm{CO}$ and residual iCORM-2, the predominant effect is CO mediated, and from a therapeutic standpoint there would not be an occasion to administer ICORM-2 alone instead of CORM2. Of interest, iCORM-2 has been demonstrated to mediate effects similar to or opposite of $\mathrm{CO}$ in vivo and in vitro in various systems. ${ }^{24,25}$

Another issue of interest was that the exposure of venoms with prothrombin activating and thrombin-like activity to CO did not eliminate coagulation in plasma despite presumed inactivation of SVMP, unlike the inhibition observed in the case of $C$. atrox and A. c. mokasen venom fibrinogenase inactivation. This partial inhibition of prothrombin activating or thrombin-like activity in these two venoms may be in part secondary to incomplete inhibition of SVMP, but also possibly secondary to CO not interacting with the SVSP also present with thrombin-like activity (Table 1). In sum, the simplest explanation for the patterns of inhibition observed was interaction of CO with SVMP catalytic sites.

A potential concern about the use of CORM-2 or other CO releasing molecules to treat SVMP mediated coagulopathy is the likelihood of the envenomed individual experiencing CO poisoning and excessive carboxyhemoglobin concentrations. This fear may be unfounded, as hundreds of animal investigations involving administration of $\mathrm{CO}$ releasing molecules as a therapeutic intervention has not in either the acute or chronic setting resulted in an adverse outcome - instead, CO releasing molecules typically reduce the injury studied. As examples, in mouse ${ }^{26}$ and rabbit ${ }^{27,28}$ models involving the administration of CORM-3 (ref. 26) and CORM-2, ${ }^{27,28}$ animals demonstrated no clinical signs of CO poisoning for days after administration of these compounds. Further, based on experiments that directly measured increases is carboxyhemoglobin concentrations in whole blood after CO releasing molecule addition, the concentration of carboxyhemoglobin would be expected to increase less than $1 \%$ in the presence of $100 \mu \mathrm{M}$ CORM-2. ${ }^{26}$ To put this information into the clinical perspective, if an individual were to smoke two tobacco cigarettes within a 90 minute period, the average carboxyhemoglobin concentrations would be on average $5 \%$, which is five times the normal concentration. ${ }^{29} \mathrm{CO}$ releasing molecules deliver CO in a site-directed manner, with even distribution throughout the solution in which they are added, permitting binding to heme or other molecular moieties on plasma proteins and within blood cells of all types. Thus, if an individual were to have systemic administration of CO equivalent to an instantaneous concentration of $100 \mu \mathrm{M}$ CORM-2, the expected increase in carboxyhemoglobin and risk of $\mathrm{CO}$ poisoning would be less than if that individual consumed one cigarette. Therefore, based on the large amount of animal studies in existence without adverse outcomes after $\mathrm{CO}$ releasing molecule exposure and the expected small amount of increase in carboxyhemoglobin expected with the concentrations of CORM-2 used in the present investigation, we would anticipate that the clinical inhibition of SVMP with CO releasing molecules would not be associated with clinical CO poisoning.

In conclusion, the present study demonstrated that $\mathrm{CO}$ inhibits SVMP fibrinogenase, prothrombin activating and thrombin-like activity from four diverse viper species. Future investigations of the effects of $\mathrm{CO}$ on other snake venoms containing metalloproteinases are warranted.

\section{Authors' contributions}

VGN designed and VGN and CMB conducted the experiments. VGN and CMB analyzed the data and wrote the manuscript. Both authors have reviewed and approve the final manuscript. This investigation was funded by the Department of Anesthesiology.

\section{References}

1 F. S. Markland Jr. and S. Swenson, Snake venom metalloproteinases, Toxicon, 2013, 62, 3-18.

2 S. H. Chiou, C. C. Hung and K. F. Huang, Characterization of a protease with $\alpha$ - and $\beta$-fibrinogenase activity from the western diamondback rattlesnake, Crotalus atrox, Biochem. Biophys. Res. Commun., 1992, 187, 389-396.

3 J. B. Moran and C. R. Geren, Characterization of a fibrinogenase from northern copperhead (Agkistrodon contortrix mokasen) venom, Biochim. Biophys. Acta, 1981, 659, 161-168.

4 R. Thakur, P. Chattopadhyay, S. S. Ghosh and A. K. Mukherjee, Elucidation of procoagulant mechanism and pathophysiological significance of a new prothrombin activating metalloprotease purified from Daboia russelii russelii venom, Toxicon, 2015, 100, 1-12.

5 G. D. Loría, A. Rucavado, A. S. Kamiguti, R. D. Theakston, J. W. Fox, A. Alape and J. M. Gutiérrez, Characterization of 'basparin A,' a prothrombin-activating metalloproteinase, from the venom of the snake Bothrops asper that inhibits platelet aggregation and induces defibrination and thrombosis, Arch. Biochem. Biophys., 2003, 418, 13-24.

6 A. K. Mukherjee and S. P. Mackessy, Biochemical and pharmacological properties of a new thrombin-like serine protease (Russelobin) from the venom of Russell's Viper (Daboia russelii russelii) and assessment of its therapeutic potential, Biochim. Biophys. Acta, 2013, 1830, 3476-3488.

7 A. V. Pérez, A. Rucavado, L. Sanz, J. J. Calvete and J. M. Gutiérrez, Isolation and characterization of a serine proteinase with thrombin-like activity from the venom of the snake Bothrops asper, Braz. J. Med. Biol. Res., 2008, 41, 12-17. 
8 K. Segers, J. Rosing and G. A. Nicolaes, Structural models of the snake venom factor $\mathrm{V}$ activators from Daboia russelli and Daboia lebetina, Proteins, 2006, 64, 968-984.

9 A. Z. Budzynski, B. V. Pandya, R. N. Rubin, B. S. Brizuela, T. Soszka and G. J. Stewart, Fibrinogenolytic afibrinogenemia after envenomation by western diamondback rattlesnake (Crotalus atrox), Blood, 1984, 63, 1-14.

10 F. G. Walter, U. Stolz, F. Shirazi, C. M. Walter and J. McNally, Epidemiology of the reported severity of copperhead (Agkistrodon contortrix) snakebite, South. Med. J., 2012, 105, 313-320.

11 F. G. Walter, U. Stolz, R. N. French, P. B. Chase, J. McNally and F. Shirazi, Epidemiology of the reported severity of cottonmouth (Agkistrodon piscivorus) snakebite, South. Med. J., 2014, 107, 150-156.

12 V. G. Nielsen and L. V. Boyer, Iron and carbon monoxide attenuate degradation of plasmatic coagulation by Crotalus atrox venom, Blood Coagulation Fibrinolysis, 2016, 27, 506-510.

13 V. G. Nielsen, D. T. Redford and P. K. Boyle, Effect of iron and carbon monoxide on fibrinogenase-like degradation of plasmatic coagulation by venoms of six Agkistrodon species, Basic Clin. Pharmacol. Toxicol., 2016, 118, 390-395.

14 V. G. Nielsen, D. T. Redford and P. K. Boyle, Effect of iron and carbon monoxide on fibrinogenase-like degradation of plasmatic coagulation by venoms of four Crotalus species, Blood Coagulation Fibrinolysis, DOI: 10.1097/MBC.0000000000000529.

15 V. G. Nielsen, Iron and carbon monoxide prevent degradation of plasmatic coagulation by thrombin-like activity in rattlesnake venom, Hum. Exp. Toxicol., DOI: 10.1177/0960327115621366.

16 V. G. Nielsen and E. Pretorius, Iron and carbon monoxide enhance coagulation and attenuate fibrinolysis by different mechanisms, Blood Coagulation Fibrinolysis, 2014, 25, 695-702.

17 V. G. Nielsen and J. I. Garza, Comparison of the effects of CORM-2, CORM-3 and CORM-A1 on coagulation in human plasma, Blood Coagulation Fibrinolysis, 2014, 25, 801-805.

18 M. Desmard, N. Amara, S. Lanone, R. Motterlini and J. Boczkowski, Carbon monoxide reduces the expression and activity of matrix metalloproteinases 1 and 2 in alveolar epithelial cells, Cell. Mol. Biol., 2005, 51, 403-408.

19 S. S. Sung and R. Hoffmann, How carbon monoxide bonds to metal surfaces, J. Am. Chem. Soc., 1985, 107, 578-584.

20 M. J. Lyle, O. Warschkow, B. Delley and C. Stampfl, Coverage and charge-state dependent adsorption of carbon monoxide on the zinc oxide (0001) surface, Phys. Rev. B: Condens. Matter Mater. Phys., 2010, 82, 165401.
21 E. D. Bloch, M. R. Hudson, J. A. Mason, S. Chavan, V. Crocellà, J. D. Howe, K. Lee, A. L. Dzubak, W. L. Queen, J. M. Zadrozny, S. J. Geier, L. C. Lin, L. Gagliardi, B. Smit, J. B. Neaton, S. Bordiga, C. M. Brown and J. R. Long, Reversible $\mathrm{CO}$ binding enables tunable $\mathrm{CO} / \mathrm{H}_{2}$ and $\mathrm{CO} / \mathrm{N}_{2}$ separations in metal-organic frameworks with exposed divalent metal cations, J. Am. Chem. Soc., 2014, 136, 10752-10761.

22 V. Telezhkin, S. P. Brazier, R. Mears, C. T. Müller, D. Riccardi and P. J. Kemp, Cysteine residue 911 in C-terminal tail of human $\mathrm{BK}(\mathrm{Ca}) \alpha$ channel subunit is crucial for its activation by carbon monoxide, Pfluegers Arch., 2011, 461, 665-675.

23 S. Chinnasamy, S. Nagamani and K. Muthusamy, $\mathrm{Zn}^{2+}$ ion of the snake venom metalloproteinases (SVMP) plays a critical role in ligand binding: a molecular dynamics simulation study, RSC Adv., 2015, 5, 70566-70576.

24 A. Jara-Oseguera, I. G. Ishida, G. E. Rangel-Yescas, N. Espinosa-Jalapa, J. A. Pérez-Guzmán, D. Elías-Vinas, R. Le Lagadec, T. Rosenbaum and L. D. Islas, Uncoupling charge movement from channel opening in voltage-gated potassium channels by ruthenium complexes, J. Biol. Chem., 2011, 286, 16414-16425.

25 O. Pak, A. G. Bakr, M. Gierhardt, J. Albus, I. Strielkov, F. Kroschel, T. Hoeres, M. Hecker, H. A. Ghofrani, W. Seeger, N. Weissmann and N. Sommer, Effects of carbon monoxidereleasing molecules on pulmonary vasoreactivity in isolated perfused lungs, J. Appl. Physiol., 2016, 120, 271-281.

26 Y. Guo, A. B. Stein, W. J. Wu, W. Tan, X. Zhu, Q. H. Li, B. Dawn, R. Motterlini and R. Bolli, Administration of a COreleasing molecule at the time of reperfusion reduces infarct size in vivo, Am. J. Physiol., 2004, 286, H1649-H1653.

27 V. G. Nielsen, N. Chawla, D. Mangla, S. B. Gomes, M. R. Arkebauer, K. A. Wasko, K. Sadacharam and K. Vosseller, Carbon monoxide-releasing molecule-2 enhances coagulation in rabbit plasma and decreases bleeding time in clopidogrel/aspirin-treated rabbits, Blood Coagulation Fibrinolysis, 2011, 22, 756-759.

28 V. G. Nielsen, M. R. Arkebauer, K. A. Wasko, S. N. Malayaman and K. Vosseller, Carbon monoxide-releasing molecule-2 decreases fibrinolysis in vitro and in vivo in the rabbit, Blood Coagulation Fibrinolysis, 2012, 23, 104-107.

29 V. G. Nielsen, D. T. Hafner and E. B. Steinbrenner, Tobacco smoke-induced hypercoagulation in human plasma: role of carbon monoxide, Blood Coagulation Fibrinolysis, 2013, 24, 405-410. 